\title{
INTERCAMBIOS CONVECTIVOS EN EL INTERIOR DE LOS EDIFICIOS
}

\author{
(CONVECTIVE EXCHANGES INSIDE BUILDINGS)
}

\author{
J. Roset ${ }^{*}$ y B. Fleury *
}

\section{RESUMEN}

La convección en el interior de los edificios es el principal mecanismo de transferencia de calor entre las partes de los mismos. El modelo numérico que se utiliza en este trabajo es bidimensional con el flujo de calor en régimen laminar. Se simulan diferentes condiciones térmicas de un cerramiento con la hipótesis de que una de sus paredes es sometida a varias distribuciones de temperatura no uniformes. Se obtienen, para estas configuraciones, los flujos de calor en las distintas partes de la pared así como la distribución final de temperaturas en el interior de toda la habitación.

\section{SUMMARY}

Convection inside buildings is the main mechanism to explain heat transfer among its parts. The numerical model, we use in this work, is bidimensional with the heat flux in laminar regime. We simule different thermal conditions of a room with one of its walls having several non-uniform distributions of temperature. We obtain, for these configurations, the heat fluxes through the different parts of the wall and the final distribution of the temperatures inside the whole room.

\section{INTRODUCCION}

La convección juega un papel fundamental en la distribución de la energía en el interior de los edificios. Este papel es aún más importante cuando se trata de edificios que utilizan los conceptos solares pasivos de captación, ya que en tal caso la convección natural es la principal responsable de la transferencia del calor desde los lugares de captación al resto de las habitaciones del edificio.

Se han ensayado tres tipos de aproximaciones diferentes para tratar el problema: modelos a tamaño real, modelización con maquetas y simulación por ordenador.

La realización de modelos a escala real es demasiado cara y difícil de generalizar como para ser rentable.

Algunos trabajos basados en correlaciones empiricas sobre modelos a escala se han realizado ya y, a pesar de que presentan dificultades importantes en cuanto a la validez de la extrapolación de los resultados a ta-

* Dept. Física. Escola Tècnica Superior d'Arquitectura del Vallès. Universitat Politècnica de Catalunya. Ap. Corr. 508, 08220 Terrasa Barcelona.

* Laboratoire des Sciences de l'Habitat. Ecole Nationale des Travaux Publics de l'Etat. Rue M. Audin. 69120 Vaulx-en-Velin. France. maño real y al alto costo de la instrumentación necesaria, se han obtenido resultados interesantes en lo que concierne a casos simples $(1,2)$.

Los modelos numéricos nos permiten una mayor versatilidad en cuanto a imponer diferentes condiciones al edificio, aunque las ecuaciones implicadas en el tratamiento de la convección natural son difíciles de tratar y nos limitan, por ahora, a casos muy simples.

El presente trabajo es un estudio numérico en el que pretendemos valorar el efecto de diferentes tipos de oberturas en una habitación.

\section{DESCRIPCION DEL CALCULO Y RESULTADOS}

Se ha realizado un cálculo sobre la resolución numérica de las ecuaciones fundamentales de la mecánica de fluidos en la aproximación de Boussinesq (3).

Para ello se ha utilizado el programa "Convec1" desarrollado en el LASH/ENTPE. Este programa resuelve las ecuaciones en dos dimensiones y en condiciones de régimen laminar, utilizando un método numérico de diferencias finitas (4). 
Las componentes adimensionales de la velocidad u y $v$, la presión $\mathrm{p}$ y la temperatura T están dadas por las siguientes ecuaciones:

$$
\begin{aligned}
& u(\delta \mathrm{u} / \delta \mathrm{x})+v(\delta \mathrm{u} / \delta \mathrm{y})+(\delta \mathrm{p} / \delta \mathrm{x})-\operatorname{Pr} \nabla^{2} \mathrm{u}=0 \\
& u(\delta v / \delta \mathrm{x})+v(\delta v / \delta \mathrm{y})+(\delta \mathrm{p} / \delta \mathrm{y})-\operatorname{Pr} \nabla^{2} v=\operatorname{RaPrT} \\
& (\delta \mathrm{u} / \delta \mathrm{x})+(\delta v / \delta \mathrm{y})=0 \\
& u(\delta \mathrm{T} / \delta \mathrm{x})+v(\delta \mathrm{T} / \delta \mathrm{y})-\nabla^{2} \mathrm{~T}=0
\end{aligned}
$$

donde $x$ e y son las coordenadas adimensionales $x^{*} / L$, $y^{\star} / L$.

Estas ecuaciones contienen dos parámetros adimensionales, el número de PrandtI

$\operatorname{Pr}=(\nu / k)$

y el número de Rayleigh (basado en la anchura de la habitación)

$\mathrm{Ra}=\mathrm{Gr} \cdot \mathrm{Pr}=\left[\mathrm{g} \beta\left(\mathrm{T}_{\mathrm{c}}-\mathrm{T}_{\mathrm{t}}\right)\left[\mathrm{L}^{3} / \nu^{2}\right] .(\nu / \mathrm{K})\right.$

donde $\mathrm{Gr}$ es el número de Grashof y en su expresión $\nu$ es la viscosidad cinemática, $\beta$ es el coeficiente de expansión volumétrica, $\mathrm{g}$ la aceleración de la gravedad, $\mathrm{L}$ la anchura de la habitación, $\mathrm{k}$ la conductividad térmica del fluido que la llena y $T_{c}$ y $T_{\text {f }}$ las temperaturas de las paredes caliente y fría respectivamente.

De forma general, podemos decir que el número de Prandtl representa la relación entre el campo de velocidades y el de temperaturas y el de Grashof entre las fuerzas ascensionales y las viscosas.

En el presente trabajo, se han estudiado siete distribuciones diferentes de temperatura superficial sobre la pared derecha de la sección de una habitación y con el resto de caracteristicas constantes: La pared izquierda es la pared caliente y es mantenida a una temperatura superficial constante e igual a $20^{\circ} \mathrm{C}$; el suelo y el techo son adiabáticos; el gas que llena la habitación es el aire; la habitación tiene una altura de 2,4 $\mathrm{m}$ y una longitud de $2,4 \mathrm{~m}$; el máximo incremento de temperatura considerado es de $5^{\circ} \mathrm{C}$.

Los números adimensionales asociados a esta configuración son:

Número de PrandtI $=0,71$

Número de Grashof $=0,96.10^{10}$

Número de Rayleigh $=0,68.10^{10}$

En las figuras Xa se presentan las isotermas en el interior de la habitación junto con las condiciones límites en temperatura sobre las paredes verticales.
En las figuras $\mathrm{Xb}$ se presentan las líneas de corriente en el interior de la habitación así como la variación del número de Nusselt a lo largo de las paredes verticales.

El número de Nusselt está definido como

$\mathrm{Nu}=(h \mathrm{~L} / \mathrm{k})$

y significa la relación entre el calor transferido $(h)$ y el que se transferiría por conducción pura a través de una capa de fluido de espesor $L(k / L)$.

La tabla I muestra los números de Nusselt globales así como los coeficientes de intercambio convectivo obtenidos ( $k=0,0258 \mathrm{~W} / \mathrm{m} \cdot \mathrm{K}, \mathrm{L}=2,4 \mathrm{~m})$ de ellos para cada configuración.

La tabla II muestra los flujos de calor por convección para las diferentes partes de la pared de cada configuración

\begin{tabular}{|c|c|}
\hline Configuración & $\varnothing$ (watts) \\
\hline $\begin{array}{ll} & \text { p.a. } \\
& \text { p.b. }\end{array}$ & $\begin{array}{l}1,95 \\
8,95\end{array}$ \\
\hline $\begin{array}{l}2 \text { p.a. } \\
\text { p.m. } \\
\text { p.b. }\end{array}$ & $\begin{array}{r}3,91 \\
3,36 \\
-0,77\end{array}$ \\
\hline $\begin{array}{l}3 \text { p.a. } \\
\text { p.m. } \\
\text { p.b. }\end{array}$ & $\begin{array}{r}3,06 \\
6,30 \\
-1,06\end{array}$ \\
\hline $\begin{array}{l}4 \text { p.a. } \\
\text { p.m. } \\
\text { p.b. }\end{array}$ & $\begin{array}{r}2,26 \\
6,94 \\
-1,54 \\
\end{array}$ \\
\hline $\begin{array}{l}5 \text { p.a. } \\
\text { p.m. } \\
\text { p.b. }\end{array}$ & $\begin{array}{r}0,95 \\
7,63 \\
-1,29\end{array}$ \\
\hline $\begin{array}{l}6 \text { p.a. } \\
\text { p.m. } \\
\text { p.b. }\end{array}$ & $\begin{array}{r}3,29 \\
7,34 \\
-4,10\end{array}$ \\
\hline $\begin{array}{l}7 \text { p.a. } \\
\text { p.m. } \\
\text { p.b. }\end{array}$ & $\begin{array}{r}3,52 \\
6,75 \\
-2,90\end{array}$ \\
\hline
\end{tabular}

Tabla I

\begin{tabular}{|c|c|c|}
\hline Configuración & Nu global & $\mathbf{h}\left(\mathbf{W} / \mathbf{m}^{2} \cdot \mathbf{K}\right)$ \\
\hline 1 & 85,2 & 0,92 \\
2 & 53,1 & 0,57 \\
3 & 63,5 & 0,68 \\
4 & 61,5 & 0,66 \\
5 & 59,5 & 0,64 \\
6 & 49,1 & 0,53 \\
7 & 55,9 & 0,60 \\
\hline
\end{tabular}

Tabla II

(p.a. $=$ parte alta, p.m $=$ parte media, p.b. $=$ parte baja $)$ 

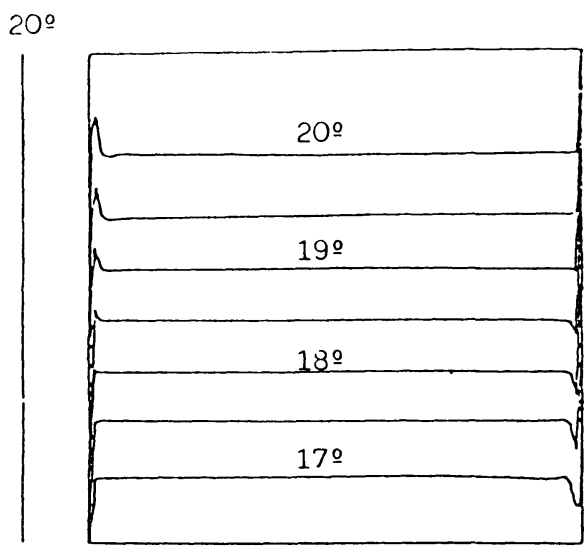

Figura 1a

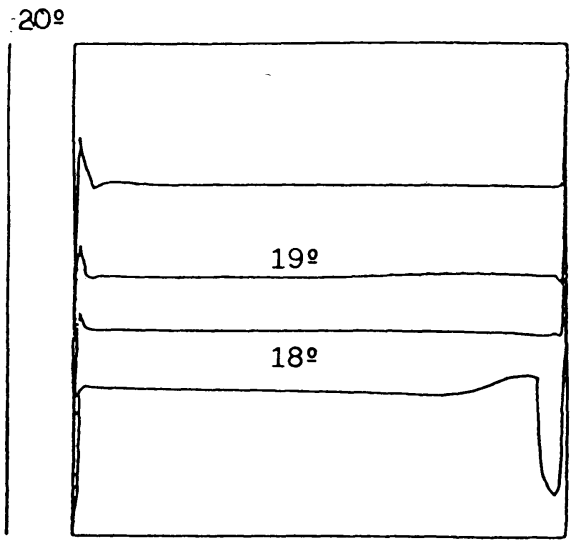

Figura $2 a$

200

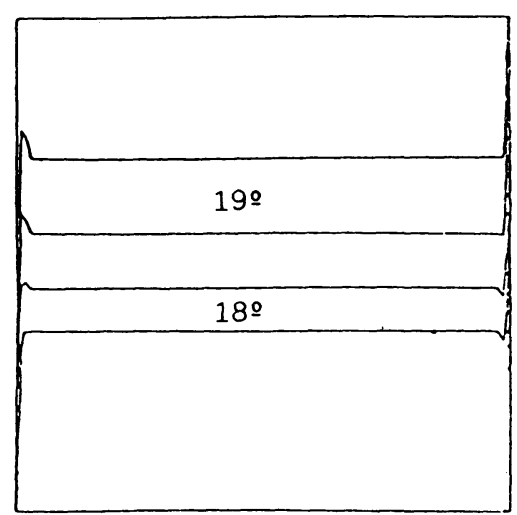

Figura 3a

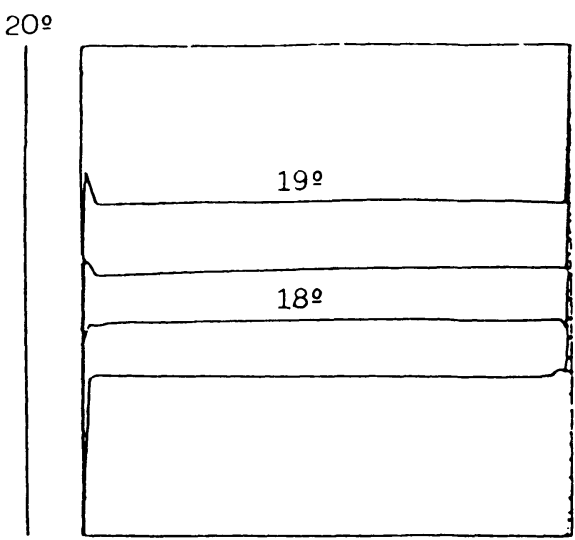

Figura 4a

(C) Consejo Superior de Investigaciones Científicas Licencia Creative Commons 3.0 España (by-nc)
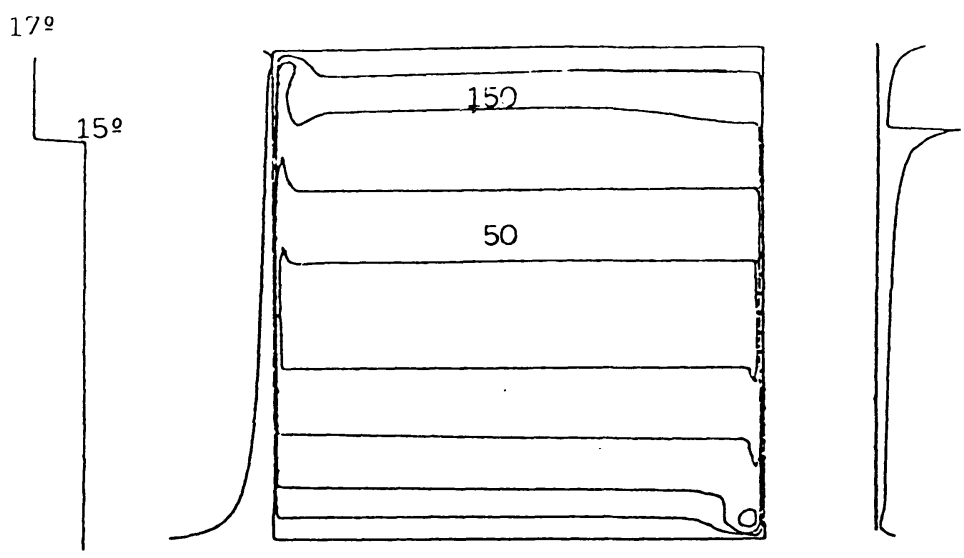

Figura 1b

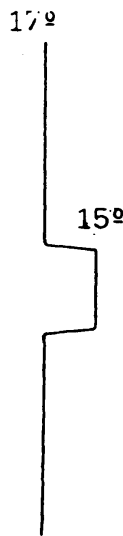

179

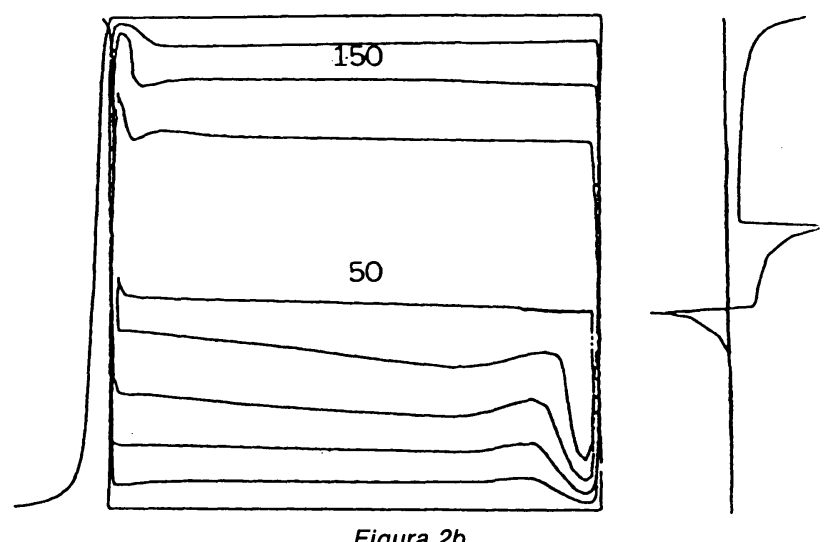

Figura $2 b$
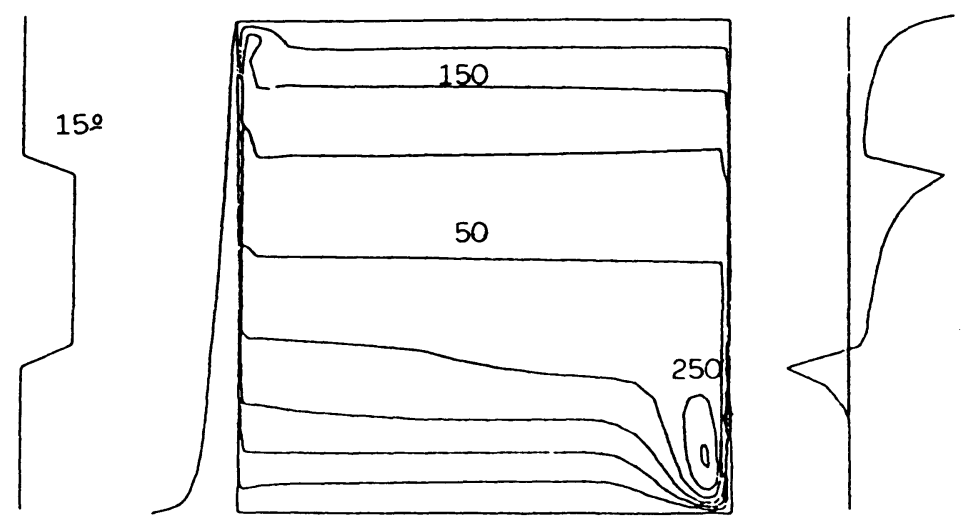

Figura $3 b$
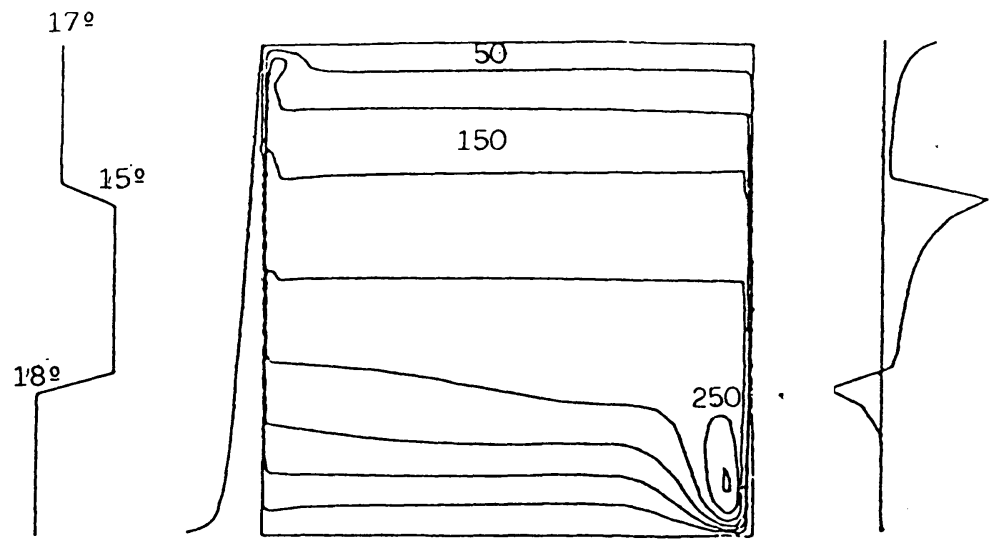

Figura $4 b$

http://informesdelaconstruccion.revistas.csic.es 


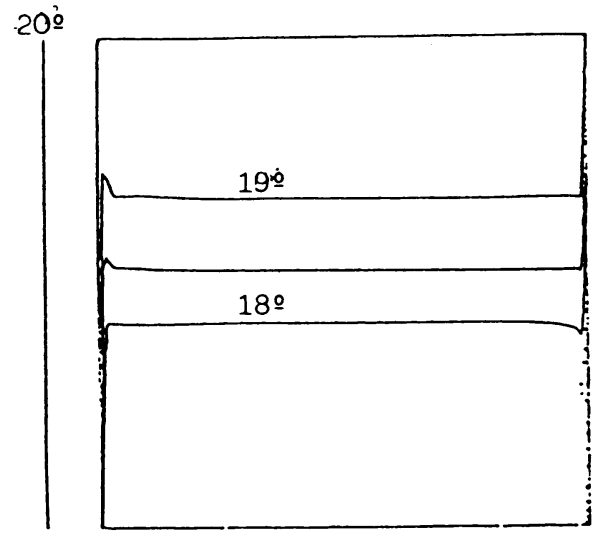

Figura 5a

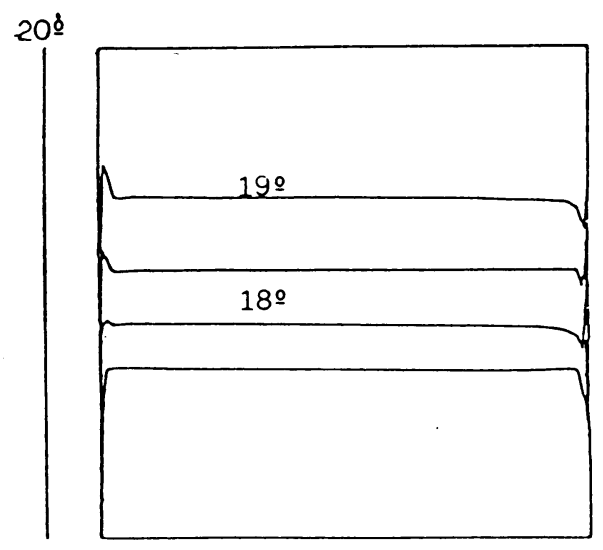

Figura 6a

2)

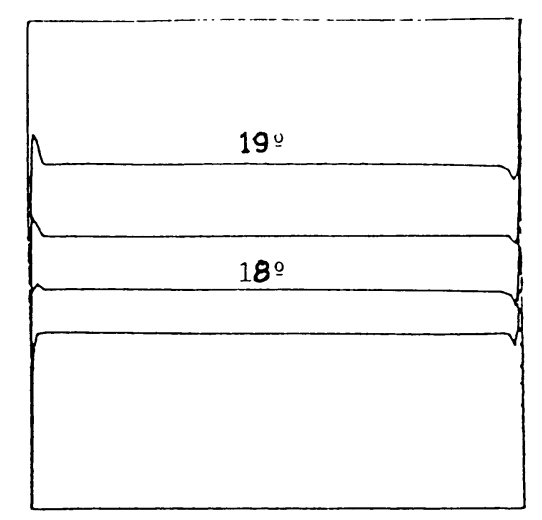

Figura $7 a$

Figuras Xa. Isotermas en el interior de la habitación sometida a unas condiciones dadas sobre las paredes laterales.

\section{DISCUSION}

En primer lugar, realizaremos una comparación entre los resultados que hemos obtenido para la pared en su conjunto (tabla I) y los parámetros utilizados por la NBECT-79 (5), para ello es necesario realizar algunos cálculos.
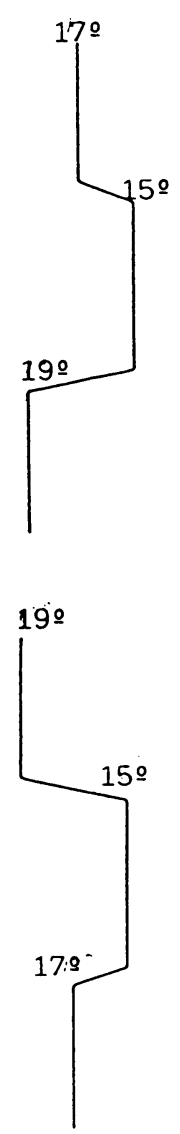

$18^{\prime}$
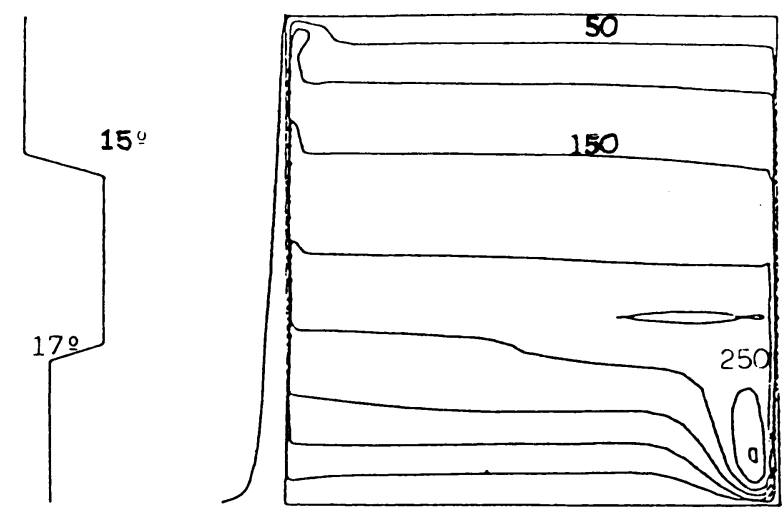

Figura $7 b$

Figura $5 b$

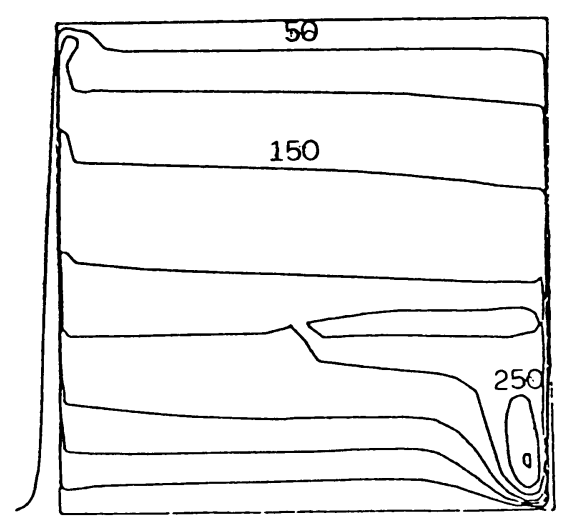

Figura $6 b$
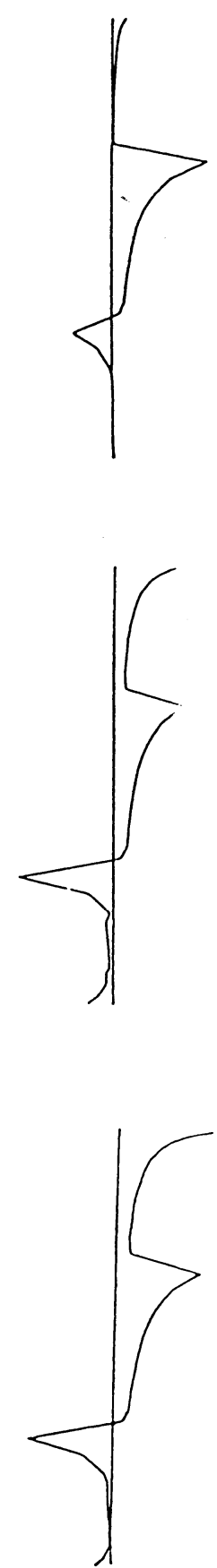

Figuras $X b$. Líneas de corriente en el interior de la habitación (escala arbitraria) y números de Nusselt locales sobre las paredes laterales.

La norma fija para todos estos casos un valor de $\left(1 / h_{1}\right)=0,11$, donde $h_{1}$ es el coeficiente superficial de transmisión de calor en el interior de un edificio.

Este coeficiente engloba tanto los intercambios convectivos como los radiactivos. A efectos prácticos acep- 
taremos que los efectos convectivo y radiactivo pueden ser desacoplados de forma que

$h_{1}=h_{c}+h_{t}$

de la comparación entre la ley de Stefan-Boltzmann:

$P=\sigma \epsilon\left(T^{4}-T_{a}^{4}\right)$

donde $\mathrm{P}$ es la potencia radiada (o absorbida) por unidad de área $\left(\mathrm{W} / \mathrm{m}^{2}\right)$

$\sigma$ la constante de Stefan-Boltzmann $=$ $5,67.10^{-8} \mathrm{~W} / \mathrm{m}^{2} \mathrm{~K}^{4}$

$\epsilon$ la emisividad del material $=0,9$ para materiales de construcción usuales

T la temperatura de la pared (grados $\mathrm{K}$ )

$\mathrm{T}_{\mathrm{a}}$ la temperatura ambiente (grados $\mathrm{K}$ )

y su aproximación linealizada

$P_{1}=h_{r}\left(T-T_{a}\right)$

donde $\mathrm{P}_{1}$ es la potencia radiada (o absorbida) por unidad de área en la aproximación lineal $\left(\mathrm{W} / \mathrm{m}^{2}\right)$.

Si aceptamos la validez de la aproximación obtenemos:

$h_{r}=\sigma \epsilon\left(T^{2}+T_{a}^{2}\right)\left(T+T_{a}\right)$

sustituyendo valores numéricos $(T=288 \mathrm{~K}$,

$\left.\mathrm{T}_{\mathrm{a}}=290,5 \mathrm{~K}\right)$ se obtiene

$\mathrm{h}_{\mathrm{r}} \approx 5 \mathrm{~W} / \mathrm{m}^{2} \mathrm{~K}$

que no varia apreciablemente para pequeños cambios de $T$ y $T_{a}$ ( $\left.\pm 2 K\right)$.

Por tanto, de (8)

$h_{c}=h_{1}-h_{r}=(1 / 0,11)-5 \approx 4 W / m^{2} K$

muy lejos de los valores, que son como máximo de 1 $\mathrm{W} / \mathrm{m}^{2} \mathrm{~K}$ presentados en el presente trabajo.

En segundo término, discutamos el comportamiento de cada una de las partes en las diferentes configuraciones. En la tabla ll, podemos observar que los flujos de calor a través de las mismas no son siempre en el sentido de dentro hacia fuera sino que en todas las configuraciones, salvo en la primera, el flujo invierte su sentido en la parte baja de la pared. Asimismo, se puede observar que una zona significativa de la mencionada parte de la pared no interviene en los intercambios convectivos (ver figuras $\mathrm{Xb}$, las zonas con números de Nusselt $\approx 0$ ).
Para explicar cuantitativamente este comportamiento hemos ensayado un ajuste de los resultados utilizando la expresión (3):

$\mathrm{Nu}_{1}=f(\operatorname{Pr}) \mathrm{Ra}_{1}{ }^{0.25}$

donde 1 es la altura de la parte considerada de la pared objeto de estudio; $\mathrm{Nu}_{1}$ es el número de Nusselt "local" de esa parte y $\mathrm{Ra}_{1}$ el número de Rayleigh "Iocal" basado en la longitud 1 y en la diferencia de temperatura

$T_{1}=T_{\infty}(y)-T_{1}$

donde $T_{1}$ es la temperatura superficial de la parte de la pared considerada y $T_{\infty}$ (y) la temperatura del aire adyacente.

Dando sus valores a los diferentes números adimensionales es fácil ver que

$h_{1}=C \cdot T_{1}^{14}$

donde $\mathrm{C}$ es una constante.

Aceptando ahora que

$h_{1}=\varnothing, / A_{1} T_{1}$

obtenemos

$\varnothing_{1}=C \cdot A_{1} T_{1}^{54}$

De la consideración de (15) y que $T_{\alpha}(y)$ en cada configuración se puede obtener como

$\mathrm{T}_{\infty}(\mathrm{y})=\mathrm{T}_{\text {apo }}+\gamma \mathrm{y}$

con $y=0$ en el centro de la pared hemos procedido al ajuste por el método de los mínimos cuadrados de los resultados del programa, obteniéndose $\gamma$ comprendidos entre 1,2 y $2,1^{\circ} \mathrm{C} / \mathrm{m}$; y $\mathrm{T}_{\text {apo }}$ entre 17,4 y $17,9^{\circ} \mathrm{C}$ como se ve en la tabla III.

El parámetro $\gamma$ es denominado, en ocasiones, parámetro de estratificación.

Tabla III

\begin{tabular}{|c|c|c|}
\hline Configuración & $\mathbf{T}_{\text {opo }}\left({ }^{\circ} \mathrm{C}\right)$ & $\gamma\left({ }^{\circ} \mathrm{C} / \mathrm{m}\right)$ \\
\hline 1 & 17,4 & 1,3 \\
2 & 17,6 & 1,6 \\
3 & 17,5 & 1,5 \\
4 & 17,6 & 1,5 \\
5 & 17,8 & 1,2 \\
6 & 17,9 & 2,1 \\
7 & 17,7 & 1,9 \\
\hline
\end{tabular}


La visualización de los flujos (ver figuras Xb) muestra una zona central inactiva rodeada por delgadas capas límite. La transferencia de calor por convección se realiza en esas capas, mientras que la zona central poco activa dinámicamente sigue el modo conductivo.

Por último, se ha considerado la posibilidad de tomar el promedio de temperaturas de las paredes que intervienen en el intercambio de calor como temperatura representativa de la habitación

$\mathrm{T}_{\text {mов }}=\Sigma_{1} \mathrm{~T}_{1} \mathrm{~S}_{1} / \Sigma_{1} \mathrm{~S}_{1}$

donde $S_{1}$ representa la superficie de cada parte de la pared.

En la tabla IV se comparan los resultados obtenidos por nuestra simulación $\left(\mathrm{T}_{\mathrm{im}}\right)$ con los obtenidos aplicando (20).

Tabla IV

\begin{tabular}{|c|c|c|}
\hline Configuración & $\mathrm{T}_{\text {im }}\left({ }^{\circ} \mathrm{C}\right)$ & $\mathrm{T}_{\text {mob }}\left({ }^{\circ} \mathrm{C}\right)$ \\
\hline 1 & 17,8 & 17,7 \\
2 & 18,2 & 18,3 \\
3 & 18,3 & 18,1 \\
4 & 18,3 & 18,2 \\
5 & 18,4 & 18,4 \\
6 & 18,6 & 18,4 \\
7 & 18,4 & 18,2 \\
\hline
\end{tabular}

\section{CONCLUSIONES}

Mediante la utilización del programa Convec 1 se ha estudiado en detalle los flujos convectivos en la sección de una habitación sobre cuya pared derecha se han analizado siete distribuciones de temperatura superficial diferentes manteniendo las restantes características constantes.

Las conclusiones más relevantes obtenidas son:

- El coeficiente de intercambios convectivos que la NBE-CT-79 supone es apreciablemente mayor que el obtenido con la simulación numérica.

- Dicho coeficiente, que es tomado como constante, se ha encontrado que depende de la diferencia de temperaturas entre la pared y el aire adyacente a ella elevada a un cuarto $\left(h_{c}^{\alpha} T^{1 / 4}\right)$.
- El intercambio convectivo se localiza en zonas muy cercanas a las paredes mientras que el resto de la habitación permanece inactivo y sigue, por tanto, el modo conductivo que lleva a la estratificación.

Asimismo, se ha considerado la posibilidad de definir como temperatura representativa de la habitación un promedio de las temperaturas superficiales de las paredes. Aunque los resultados obtenidos son buenos, creemos que no podemos establecer conclusiones en este punto, ya que la temperatura del aire de que partíamos era de $17,5^{\circ} \mathrm{C}$ y el máximo incremento considerado de $5^{\circ} \mathrm{C}$ (entre $15^{\circ} \mathrm{C}$ y $20^{\circ} \mathrm{C}$ ) por lo cual tampoco se podian producir grandes separaciones entre los valores de $T_{\text {mob }}$ y $T_{\text {im }}$. Por este mismo motivo, no creemos tampoco poder establecer conclusiones sobre otros factores que afectan al confort como son el gradiente cabeza-pies o la asimetría de la temperatura radiante.

Actualmente se está trabajando en el programa para intentar aumentar la rapidez del mismo y garantizar su convergencia en la simulación de incrementos de temperatura mayores. Una vez conseguido lo cual, estaremos en condiciones de discutir con mayor profundidad las cuestiones relacionadas con el confort en el interior de los edificios.

\section{AGRADECIMIENTOS}

Uno de nosotros (J. R.) agradece a la Comissió Interdepartamental per a la Recerca i Innovació Tecnològica (CIRIT) la concesión de la ayuda económica necesaria para la realización de este trabajo.

\section{REFERENCIAS}

(1) D. D. WEBER. Similitude Modeling of Natural Convection Heat Transfer Through an Aperture in Passive Solar Heated Buildings. LA-8385-T. Thesis UC-59c. 1980.

(2) J. D. BALCOMB, K. YAMAGUCHI. Heat Distribution by Natural Convection. Eighth National Passive Solar Conference. Santa Fé, New Mexico. September 5-10, 1983.

(3) A. BEJAN. Convection Heat Transfer. John Wiley and Sons. 1984.

(4) A. GADGIL. On Convective Heat Transfer in Buildings Energy Analysis. Ph. D. Thesis, L.B.L. report 10900, May 1980.

(5) NBE-CT-79. 